Article

\title{
Sustainable City: Energy Usage Prediction Method for Electrified Refuse Collection Vehicles
}

\author{
Rui Zhao ${ }^{1}$, Tudor Stincescu ${ }^{1}$, Erica E. F. Ballantyne ${ }^{2, *}$ ib and David A. Stone ${ }^{1}$ \\ 1 Department of Electronic and Electrical Engineering, The University of Sheffield, Sir Frederick \\ Mappin Building, Mappin Street, Sheffield S1 3JD, UK; r.zhao@sheffield.ac.uk (R.Z.); \\ tstincescu1@sheffield.ac.uk (T.S.); d.a.stone@sheffield.ac.uk (D.A.S.) \\ 2 Sheffield University Management School, The University of Sheffield, Conduit Road, Sheffield S10 1FL, UK \\ * Correspondence: e.e.ballantyne@sheffield.ac.uk; Tel.: +44-114-222-3378
}

Received: 28 August 2020; Accepted: 18 September 2020; Published: 21 September 2020

check for updates

\begin{abstract}
With the initiative of sustainable smart city space, services and structures (3S), progress towards zero-emission municipal services has advanced the deployment of electric refuse collection vehicles (eRCVs). However, eRCVs are commonly equipped with oversized batteries which not only contribute to the majority of the weight of the vehicles but also remain a consistent weight, independent of the stage of charge (SoC), thus crucially jeopardising the significance of eRCVs in sustainability and economic strategies. Hence, customising the battery capacity in such a way that minimises its weight while storing ample energy for stalwart serviceability could significantly enhance its sustainability. In this study, taking only addresses as input, through an emergent two-stage data analysis, the energy required to collect refuse from a group of addresses was predicted. Therefore, predictions of the battery capacity requirement for the target location are possible. The theories and techniques presented in this paper were evaluated using real-life data from eRCV trials. For the same group of addresses, predicted results show an averaged error rate of $8.44 \%$, which successfully demonstrates that using the proposed address-driven energy prediction approach, the energy required to collect refuse from a set of addresses can be predicted, which can provide a means to optimise the vehicle's battery requirement.
\end{abstract}

Keywords: eRCV; route planning; energy prediction

\section{Introduction}

As shown in the statistics published on March 2020, greenhouse gas (GHG) emissions from the UK transportation sector accounted for over 27 per cent of all UK GHG emissions [1], while the petroleum burned in internal combustion engines (ICEs) accounted for around 43 per cent of GHG emissions from the overall figure of the year 2019 [1]. Although refuse collection vehicles are only a limited proportion of the 0.5 million heavy goods vehicles (HGVs) registered in Great Britain, the significance of GHG emissions from HGVs has been amplified due to the large increase in new battery-electric cars [2]. Recent studies show that UK households generate over 28 million tonnes of waste yearly, along with other waste, most of which is sent to incinerators or landfills or used for recycling [3]. However, regardless of these waste management methodologies, the reverse supply chain relies on the collection and transportation of waste, which rely heavily on refuse collection trucks. In 2017, the demand for new supplementary refuse collection vehicles (RCVs) increased by more than 20 per cent [4].

Diesel-fuelled internal combustion is a tried and tested vehicle propulsion technology, used by waste collection vehicles across the UK and around the world. However, with research demonstrating the importance of sustainability in achieving a smart city [5], the progress towards net-zero greenhouse gas emissions [6] necessitates its demise. Like any typical HGV, an RCV's energy consumption and 
corresponding emissions are significant. Current literature shows that an RCV can consume over 540 million litres of fossil fuels per year [7]. In conjunction with considerably high energy consumption, tailpipe emissions also have proven contributions to global hazardous gas accumulation. With their support for sustainability as well as international agreements including the Paris Agreement of 2015, both the UK and other major powers have been committed to reducing their GHG emissions [8]. In order to reduce the environmental impacts introduced from municipal waste collection, RCVs powered by alternative energy sources, such as compressed natural gas (CNG) and hybrid hydraulic systems, have been introduced. Although the deployment of CNG-RCVs in the US is rising [9], RCVs in the UK are still rarely powered by natural gas. However, literature shows that the tailpipe emission of a CNG-RCV is, in fact, higher than a diesel RCV, since the fuel efficiency of a CNG engine is significantly lower than a diesel-powered internal combustion engine (ICE) [10]. Further, the escaped and unburned natural gas from both the CNG fleet and refuelling infrastructure also contributes a large amount of GHG emissions as the global warming potential of natural gas is 34 times higher than that of carbon dioxide [11]. Considering that each CNG refuelling station can only support 20-30 RCVs, has its own environmental impacts and may have leakages or other forms of pollution $[10,12]$, the environmental benefits, from the GHG reduction point of view, are scant. Greater than $70 \%$ of a hydraulic hybrid RCV's braking energy can be recovered [13]; however, its energy is still sourced initially from the combustion of fossil fuels.

As a means of driving a vehicle, electric motors have an extended history compared with ICEs. Over the last decade, there has been a significant increase in the adoption of electric vehicle (EV) powertrains $[12,13]$. These are shown to be less polluting with a smaller carbon footprint when compared to their traditional ICE counterparts, and the growth of renewable energies means that the GHG emissions relating to electricity generation are dwindling. In addition to its environmental benefits, the electric refuse truck could also offer significant financial benefits. Published articles indicate that operating electric refuse truck fleets could produce a daily fuel reduction of 100,000 litres for waste management operators with 800 vehicles [14], and the economic significance may become increasingly apparent with higher levels of fossil fuel prices [15]. The Department for Energy and Climate Change, UK, predicts that the mean fuel price will be increased by at least 22 percent from 2012 through 2030 [16], whilst other reports also indicate the prices of fossil fuels continuing to rise in the near-term [17]. In addition to a reduced operation cost, the costs of initial infrastructure such as charging stations will also become lower, further emphasising the cost benefits of operating electric-powered RCV fleets.

However, large scale application of electric refuse collection vehicles (eRCVs) remains rarely seen on the market. On the one hand, an all-electric RCV can be three times the price of a conventional diesel RCV [10], mainly caused by its expensive battery pack and corresponding management system. On the other hand, worldwide research shows that an electric vehicle is generally four times the weight of a similar ICE vehicle, mainly due to its onboard energy storage components. Therefore, a considerable amount of energy is consumed to operate the RCV itself to maintain traction [10]. Hence, for an electric waste truck, optimising and reducing the weight of its onboard energy storage system could significantly improve its fuel economy while reducing its initial procurement cost.

When operating a fleet of conventional diesel-power RCVs, a waste management operator could control the amount of diesel refuelled in advance of the collection round to minimise the energy wasted caused by carrying superfluous fuels. Considering vehicle fuel tanks are commonly constructed from thin layers of metal or polyethene [18], their weight is relatively low when compared to their contents. These characteristics provide the flexibility and efficacy to optimise the kerb weight of an RCV by adjusting its fuel level. However, when operating a fleet of electric waste trucks, this strategy no longer applies. Firstly, electric collection vehicles use secondary batteries such as lithium batteries as a replacement technology to deliver energy [19], which means the weight of the energy storage system (battery) is permanent, therefore pre-determining the kerb weight of an eRCV that cannot be adjusted by changing its "fuel" level (the state of charge). Secondly, the modern vehicle battery pack is 
internally modularly designed using industrial standard battery cells [20], which naturally provide customer designed battery capacity without profoundly affecting the ease of manufacture.

However, in order to moderately optimise the battery capacity, the minimum energy required to operate the desired collection service is preconditioned. Even though pre-defined driving cycles are commonly used for measuring tailpipe emissions and fuel economy of small-sized passenger cars [21], the waste collection vehicle, commonly working under conditions such as low speed and frequent stop-start cycles, is expected to produce inconsistent results when tested or simulated under these driving cycles. Meanwhile, street characteristics and urban terrain have been recognised as additional critical factors for driving energy calculation [22]. These characteristics trends are distinctive among cities; thus, definitive studies and calculations are preferred. Although research has demonstrated the advantage, technology and strategy of utilising sensors to collect data for analytical purposes [23], the nature of this proposed energy prediction restricts the methodology to being static data driven, since it is a solution that relies on addresses and GIS databases.

In order to effectively analyse the energy usage of a desired eRCV fleet, in this paper, comprehensive analyses and calculations, driven by both geographic data and collection addresses, are presented. The approach proposed in this paper can be classified into two stages, converting addresses to a simulated drive route and then calculating the energy usage of this simulated route. To begin with, a methodology is proposed for producing a simulated driving route, representing an electric collection truck's departure from the depot, the collection from a given group of addresses, and finally the drive back to the depot. Next, a vehicle designated model, which calculates the energy usage from the previously generated drive route, is outlined. By combining the algorithm of route generation with the model of energy usage calculation, this paper demonstrates how the exact amount of energy to collect from a given set of address is predicted. The paper also contains a brief overview of modern route-finding and vehicle modelling techniques specific to electric vehicles.

\section{Materials and Methods}

\subsection{Current Collection Vehicle Development and Electric Transition}

In cities like London, there are more than 500 collection vehicles on the road every day [24], operated by local councils and waste management enterprises. An RCV generally operates a low-speed duty-cycled profile on restricted residential areas, and together with the onboard equipment used for bin lifting and waste compression, the energy consumption is considerably high [9].Due to its frequent start-stop manoeuvres, low average speed and considerably high payload, the RCV consumes significantly more energy even compared with a conventional heavy goods vehicle. A case study for the Royal Borough of Greenwich's fleet shows that a 26 ton diesel powered refuse truck, when operating in an urban environment, consumes up to nearly 1 litre of diesel per kilometre [25]. Furthermore, those products of combustion, such as carbon dioxide and nitrogen oxides, lead to substantial environmental influences. When utilising fossil fuels, such as petroleum diesel, which is constructed by alkanes, cycloalkanes and aromatic hydrocarbons, up to 25 carbon atoms per molecule, a significant amount of carbon dioxide is exhausted as the primary product from the oxidation reactions [26]. In the meantime, the burning of aromatic hydrocarbons also produces gases such as nitrogen oxide which contribute to poor air quality. Furthermore, the combustion also produces particulate matter that may also be a primary pollutant in urban areas. Research shows that long-term exposure to NOx and PM2.5 could result in reduced life expectancy among the public [27].

Refuse collection has a variety of structural advantages, making it suitable for electric transition, especially when compared with other heavy transportation or collection vehicles. On the one hand, an electric RCV that is purely powered by battery technology does not generate tailpipe emissions, thus keeping pollution away from the urban area. On the other hand, its frequent start-stop nature can be easily achieved when driven by electric motors. The instant torque can provide extra control when starting, while the braking energy can be easily regenerated by charging the battery. The European 
Commission is working dynamically in promoting the adoption of new energy-efficient vehicles, including eRCVs. In the UK, some waste management operators have begun the electrification process to convert their conventional RCV fleets. A local London council has started to trial eRCVs and has been swapping their in-service vehicles in a variety of locations [28]. Other major councils such as Sheffield, Greenwich and Westminster are have also planned or commissioned their own experimental eRCV fleets $[25,29,30]$.

However, refuelling a diesel RCV clearly requires far less time than charging an eRCV. In order to dispel the range anxiety as well as demonstrate robust serviceability, most of those eRCVs are fitted with a large battery, resulting in a vehicle that is able to double-shift without recharging. For example, the battery pack of the experimental eRCV deployed in the Royal Borough of Greenwich is able to power the vehicle for a 14-h shift [31]. However, this requires larger batteries, which causes additional energy consumption during the standard collection period and significantly increases the procurement cost.

\subsection{Research on Energy Prediction}

There are several existing methods for predicting energy consumption. Some of them are traditional, heuristic approaches based on computing instantaneous energy usage (power) and adding the results over time. Newer solutions have a higher degree of novelty and include metaheuristic algorithms used in the context of combinatorial optimisation, such as particle swarm optimisation and Bellman-Ford.

The first and most commonly used method is the coulomb count method [32], also known as the current integration method, which takes advantage of the exponential time-dependency of charge with respect to current and states that electric charge is the time integral of current.

The main drawback of this method is that due to the continuous nature of the integration operation, the measurement cannot be reset within the simulation. Other more complex methods exist; however, for the purpose of this simulation project the coulomb counting method was deemed sufficient and was successfully integrated in the software drivetrain model.

Another commonly used method is the voltage monitoring approach [32]. It converts instantaneous measured voltage to relative state of charge (SoC) based on a specified voltage-SoC discharge curve of the battery. However, the voltage-SoC relationship is usually loosely defined compared to the dependency between voltage and current or temperature. This can be countered by regularly applying a correction factor to the simulation, but nonetheless estimations based on this method tend to be less accurate as variations in the voltage-SoC curve tend to be very small in the optimal battery operation range.

Combinations of the previously presented methods exist, and have been successfully implemented in systems, but the combined approach also requires the modelling of a decision factor that is able to switch between the predictions generated by the two approaches. A Kalman filter has been proven to be a good decisional factor, thanks to its ability to control a "trust" factor that can switch between predictions [33,34].

Other metaheuristic techniques have been applied to the energy use prediction problem with varying degrees of success. One study suggests that the more flexible the algorithm, the more capable it is of fitting the model equation system [35].

\subsection{Research on Route Prediction}

While most frequently chosen algorithms towards automated route plan development such as travel distance sensing, $\mathrm{A}^{*}$ or Dijkstra-derived methods usually perform in a robust manner, generating solutions that are right most of the time, they can be further improved in the context of route planning for electric vehicles, particularly given their different driving characteristics compared to traditional ICE powertrains, such as instant maximum torque delivery and limited range that is significantly influenced by road and outside weather features [36]. 
Existing research describes novel methods in energy-efficient routes specifically tailored for EV cars given several data points, ranging from simple heuristic algorithms to metaheuristic touching AI territory capabilities. Several applied mathematical methods have been found, considered and applied to an EV car context, with varying degrees of success depending on the degree of algorithm flexibility and complexity. Applied to a force-based, first-order vehicle model similar to that previously described, methods such as particle swarm optimisation (PSO), a variation of the simple bee algorithm, have been found to decrease energy usage by $9.2 \%$ compared to a Google Maps route, opening up entirely new research strategies in the field, potential directions ranging from improving the PSO algorithm to introducing and applying new algorithms to the presented problem [37]. Similarly, other optimisation approaches like the ant colony algorithm exhibit abilities to optimise solutions to the problem given enough calculation factors [38]. A Tabu Search-based solution has also been considered, and due to its flexibility in problem solving it has been found to be a robust solution for solving similar routing problems [39]. Finally, a Bellman-Ford approach has been attempted, and while the method works well with small-scale data, performing consistently towards finding the optimum problem solution, it is not a consistent method for solving large-scale data. One solution to this may consist of map pre-processing, although this may be impractical depending on the size of the map. Finally, the metaheuristic techniques described in other papers written by the same authors yield better results, although they are slower to process on small data (maps) but have higher degrees of accuracy [35].

\section{The Methodology of Address-Driven Energy Prediction}

Conventionally, a local council holds a large number of locations to be visited for waste collection. With the expanding sprawl of the urban area increasing the number of locations that need to be visited, the collection schedule is becoming more solidified. When introducing electrical waste trucks to the collection fleets, the risk of service interruption is therefore of major importance. As illustrated in [28-30], empirical data presented in various articles demonstrate that most eRCV providers were advancing a large, in terms of capacity, battery as the credential for robust service availability. However, the weight of eRCVs' onboard energy storage device, the battery, is not proportional to the amount of energy stored, and carrying an over-sized battery could significantly undermine the eRCV's advantage of sustainability. In this section, a methodology is presented to model the energy utilisation of an eRCV, thereby predicting the energy usage for any given refuse collection route, the overall objective being to determine the optimal SoC strategy for each vehicle, with a view to reducing the battery capacity and thus reducing the vehicle's kerb weight. The result could also contribute to lowering overall costs associated with eRCVs and predicting available battery capacity at the end of the collection day, for example, for grid support.

Through an emergent address analysis process, the schedule address book for a given day provided by the local authority waste management division can be converted into a dataset that is classified with the catalogue of roads. This dataset consists of all geographic coordinates, i.e., longitude, latitude and altitude, correlating to exact locations of the bins to be collected. In order to gain a complete route that is able to be used for the total energy usage calculation, the depot of the RCV fleet is also added and set to be the origin and termination point for the collection route. Based on these addresses and coordinates, together with other requirements and regulations, such as the RCV only being able to collect bins on a downhill cycle, an imitated route that potentially represents a real-world driving route can be generated. This generated route has an excellent time-domain resolution and envisions the states of the vehicle, and furthermore it is able to visualise a simulated speed, distance and elevation at each second of the simulated route, hence providing the same data as the data recorded from a satellite positioning-logging device installed on a collection truck. With the information provided by the virtual route data, a novel vehicle model solution was implemented, which shows the energy drained through the cruise and collection activities from the battery, through electronic power converters of the vehicle, to the main drivetrain and other auxiliary components such as the garbage compactor and in-cab air conditioner. The simulated energy figure focuses on both the overall usage of a given route and 
instantaneous power consumption in a one-second average interval. A simplified system topology is shown in Figure 1, which indicates its three main subsystems: address decode, route simulation and energy usage prediction. In the following section, a detailed theory of operation is introduced.

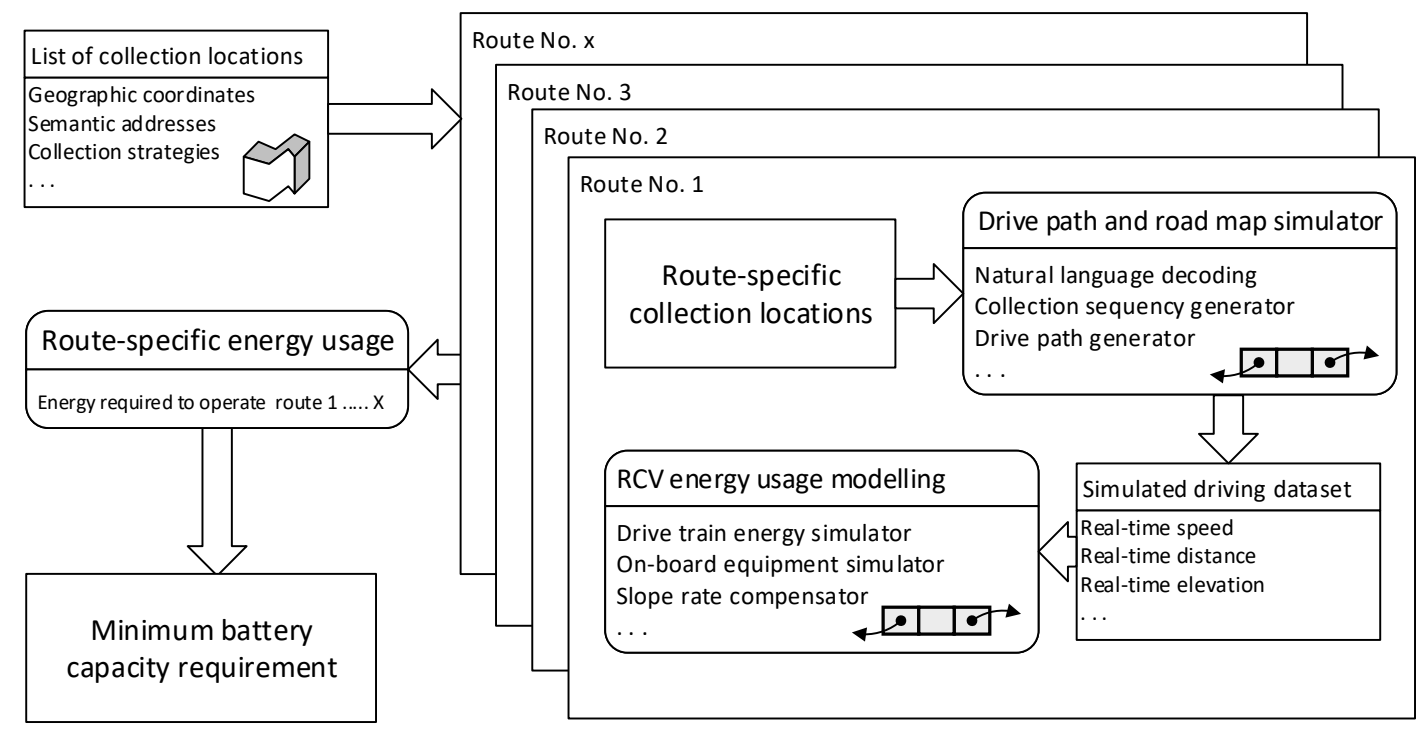

Figure 1. System topology of the proposed address-driven energy prediction methodology.

Utilising this methodology, all planned address groups can be analysed and produce a cluster of figures that hold energy usage data referring to the theoretical energy required to collect refuse from each group of addresses. Based on these route-level energy figures, optimisation of the onboard battery can therefore be conducted. First, through re-constructing the address group, the route with salient energy consumption can be modified. Secondly, a dedicated battery pack can be designed so that its stored energy can match the requirement of the waste management operator while maintaining a limited weight.

\subsection{Driving Path Simulation}

As introduced in the previous sections, the first stage of the proposed address-driven energy prediction approach is generating a simulated virtual route from the addresses which a collection vehicle is scheduled to visit. The data source of the simulated drive route is the collection rota, used by drivers of the in-service diesel $\mathrm{RCV}$, obtained from the local authority waste management division. The rota, as grouped by collection rounds, details each address to be collected with both geographic information and addresses written in natural language. The data structure of the rota is shown in Table 1.

Table 1. Data structure of the rota provided by the local authority.

\begin{tabular}{ccc}
\hline Item & Format & \\
\hline Round & Integer & A number used to mark each collection run, e.g., 00000001 \\
Address & String & Address in natural language, e.g., Number 10, ABC Road, London \\
Postcode & String & Exact postcode of the address showing above, e.g., AB1 2CD \\
Roundgroup & String & Indication of which day of the week this round will operate, unused \\
Latitude & Float & Latitude of the address \\
Longitude & Float & Longitude of the address \\
\hline
\end{tabular}

With a rota following the structure shown in Table 1, the address to be visited by a given vehicle at a given day can be identified. Next, the "Address" string of each address is analysed using a simplified natural language processing (NLP) engine. While processing each address, its house number and the 
street of the address are decoded; therefore, all addresses are grouped by street name. The address data to be processed are written in a loosely structured pattern with house number and street name included in the string. Hence, in contrast to a conventional genetic six-step NLP engine, only the content determination, text structuring and referring expression generation are being processed, while the sentence aggregation, lexicalisation and linguistic realisation are trimmed out. During this process, a limited number of addresses are spread out due to difficulties in finding the house number and/or street name; such examples could be addresses similar to "The end of the road, ABC street". In order to decode those addresses, a geo-location decoder is implemented. By querying their latitude and longitude data from a geographic information system (GIS) information provider, such as Google Maps, these uncertain addresses can be approximated. Although a query may only return its nearby house number, a negligible effect is observed from the final result, since an RCV will stop for a cluster of refuse bins, instead of at each bin location.

Due to the continuity of house numbers for each street, the extremum is identified and followed by its elevation data requested from the GIS system, where the starting and finishing location of a collection street is identified. Next, the finishing point of a collection street is used as an origin, and the next starting point in another collection street with minimum cruise distance is located, keeping the driving path at a minimum length. This process is recursive, until all streets within a route are processed, therefore generating a set of driving segments which could make up the whole virtual driving route when linked together. For each segment, a driving path that follows the actual road map is therefore generated, which represents a real-world driving path an RCV could follow. After connecting all segmental paths as well as the path between the depot and collection area, the complete driving route can be produced.

Based on this virtual driving route, a novel trapezoidal approximation approach is implemented so that a second-by-second distance and speed dataset is created. During the collection stage, this trapezoidal approximation assumes an RCV is operating on a pre-defined start-stop interval, i.e., it will stop after a given distance to empty a cluster of bins. Initially, the algorithm simulates the acceleration of the RCV with a pre-set positive slope, from stationary, until the speed reaches a pre-defined maximum value. At the end of each second, the simulated vehicle speed and travel distance are recorded. Then, the algorithm puts the vehicle into constant speed driving until the remaining distance is just enough to complete deceleration, while also recording the simulated speed and travel distance according to the same period. Finally, the algorithm simulates the deceleration stage, with a pre-set negative slope, from its max speed to 0 . Then, a zero-speed interval with random length with a pre-set interval is added, which represents the driver emptying the bins before initialising the next cycle. During the cruise section from one street to the other, this approach assumes that the RCV stops at each traffic light for a pre-defined period. Therefore, the process is similar to the collection stage but with different speed and distance factors.

The outcome from the proposed algorithm is a dataset similar to a recorded dataset from an onboard satellite positioning-logging device. Hence, it can be used as the input of the energy consumption model and calculate the energy required to collect from this group of addresses.

\subsection{Energy Consumption Modelling}

The presented vehicle model simulates a battery-electric vehicle (BEV) heavyweight powertrain that has been adapted to reflect the performance and behaviour of an eRCV during a normal refuse-bin collection routing operation. Key capabilities include the ability to account for the behaviour of the auxiliary vehicle power systems, such as heating, cooling and the refuse compression system. It also approximates the effect of road slope increase on power usage. The model is also capable of simulating energy harvesting through braking.

The proposed simulation solution was initially developed in MATLAB using the related Simulink and SimScape versions in the base workspace and environment. Several model approaches were developed in order to estimate different capabilities of various simulation blocks within Simulink. 
After careful consideration, the most promising design in terms of versatility and ease of use was chosen. The final model, as shown in Figure 2, was developed starting from a multi-level power source drivetrain model available in the Simulink SimScape library. Compared to basic Simulink blocks for which the output can be seen only if an observer block is attached to the signal line (e.g., scope, bar, final value). SimScape allows for complete parameter observation thanks to the Solver Explorer application. Here, time-based evolution of all system and subsystem parameters can be seen down to a library-defined block level. This also allows for a much quicker and better understanding of the model.

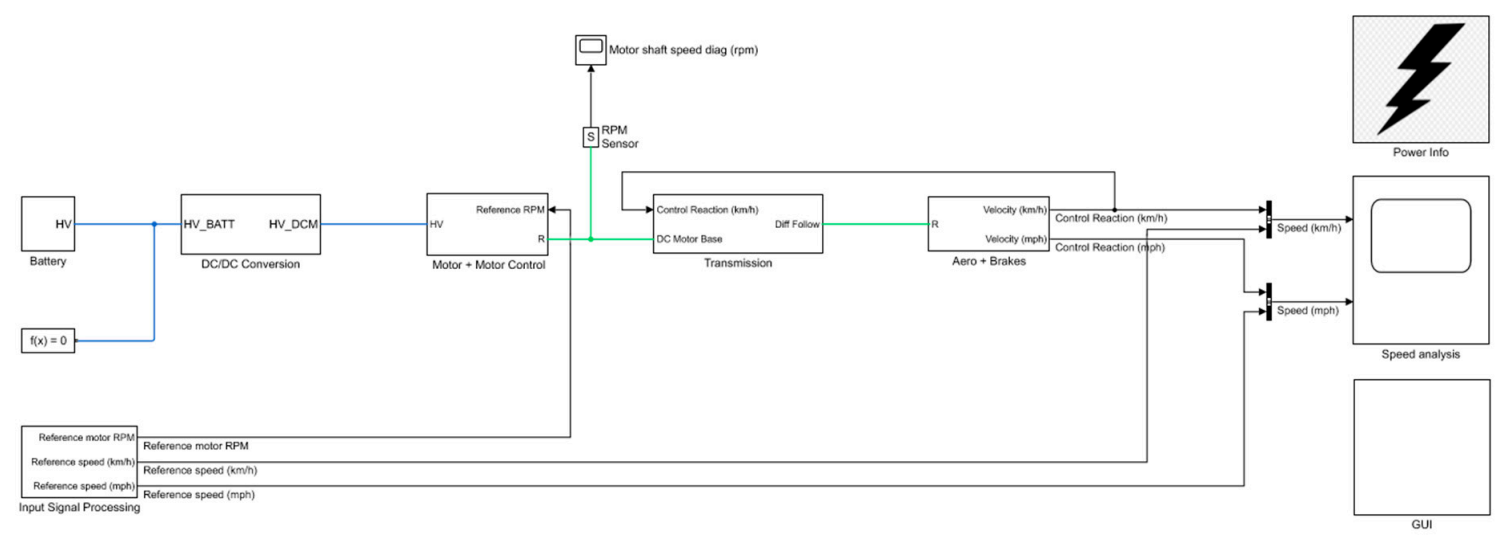

(a)

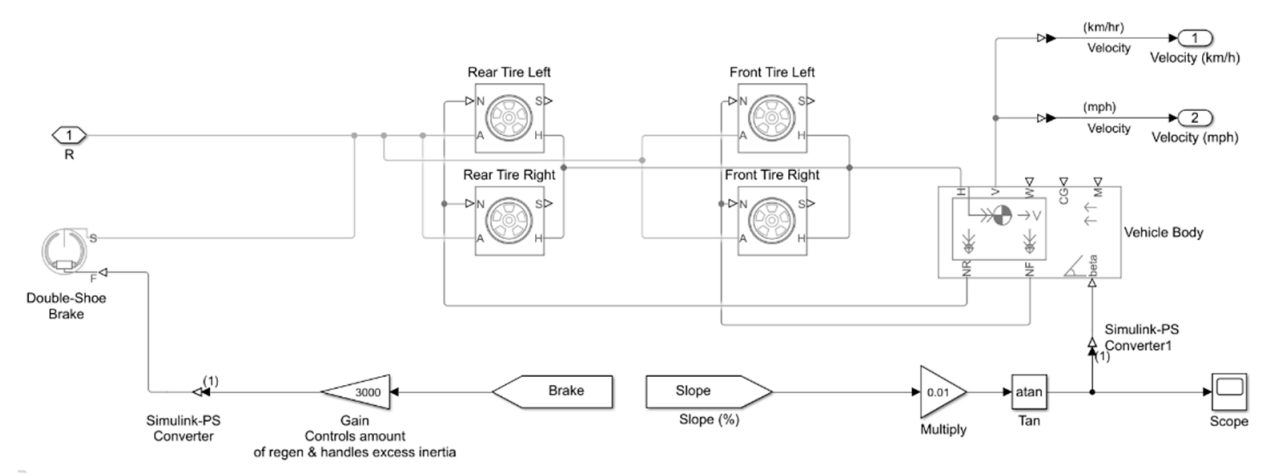

(b)

Figure 2. Electric refuse collection vehicle (eRCV) software model, (a) model topology, (b) detailed block diagram showing the aero and break sub-module.

The chosen approach consists of a simple unidirectional model that feeds input data into the system and outputs iteratively with respect to the time step choice. The input signal processing block adapts input data which is then fed into the direct current (DC) motor control module. Then, the block outputs the power demand to the motor which in turn draws the required energy from the battery model through the DC/DC conversion module. Additionally, the DC motor block also outputs mechanical rotational movement parameters to the other side of the model. The gearbox and transmission block ratios the DC motor output accordingly, then feeds in the resulting parameters to the aerodynamics subsystem. Here, the control module applies any required braking and takes the block output as the system reaction to the input.

Finally, key simulation information is outputted to a number of plot monitors, grouped with respect to monitoring areas. The speed analysis scope block shows the evolution in time of two-speed values, the input dataset and the model's speed that is mapped and corrected by the control module to the former input. The block is capable of showing the speed-time performance in both $\mathrm{km} / \mathrm{h}$ and mph using linear conversion, as seen in Figure 3. Furthermore, this block is useful in diagnosing any problems that may arise with the system's controlled response. 


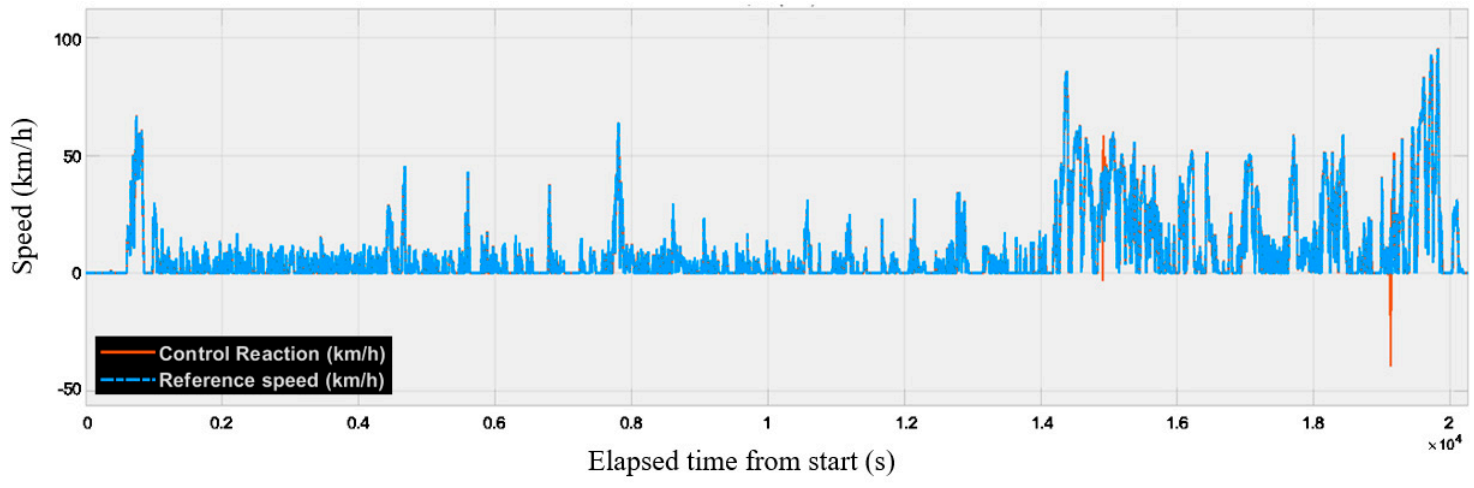

Figure 3. Vehicle speed control monitoring.

Similarly, the power info block consists of several scope blocks that measure mechanical and electrical power-related parameters that are also useful for diagnosing any problems that may arise, such as motor shaft revolutions per minute (RPM), instantaneous battery losses and absolute power deployment/harvest as functions of time that synchronise with the speed analysis scope, as presented in Figure 4.
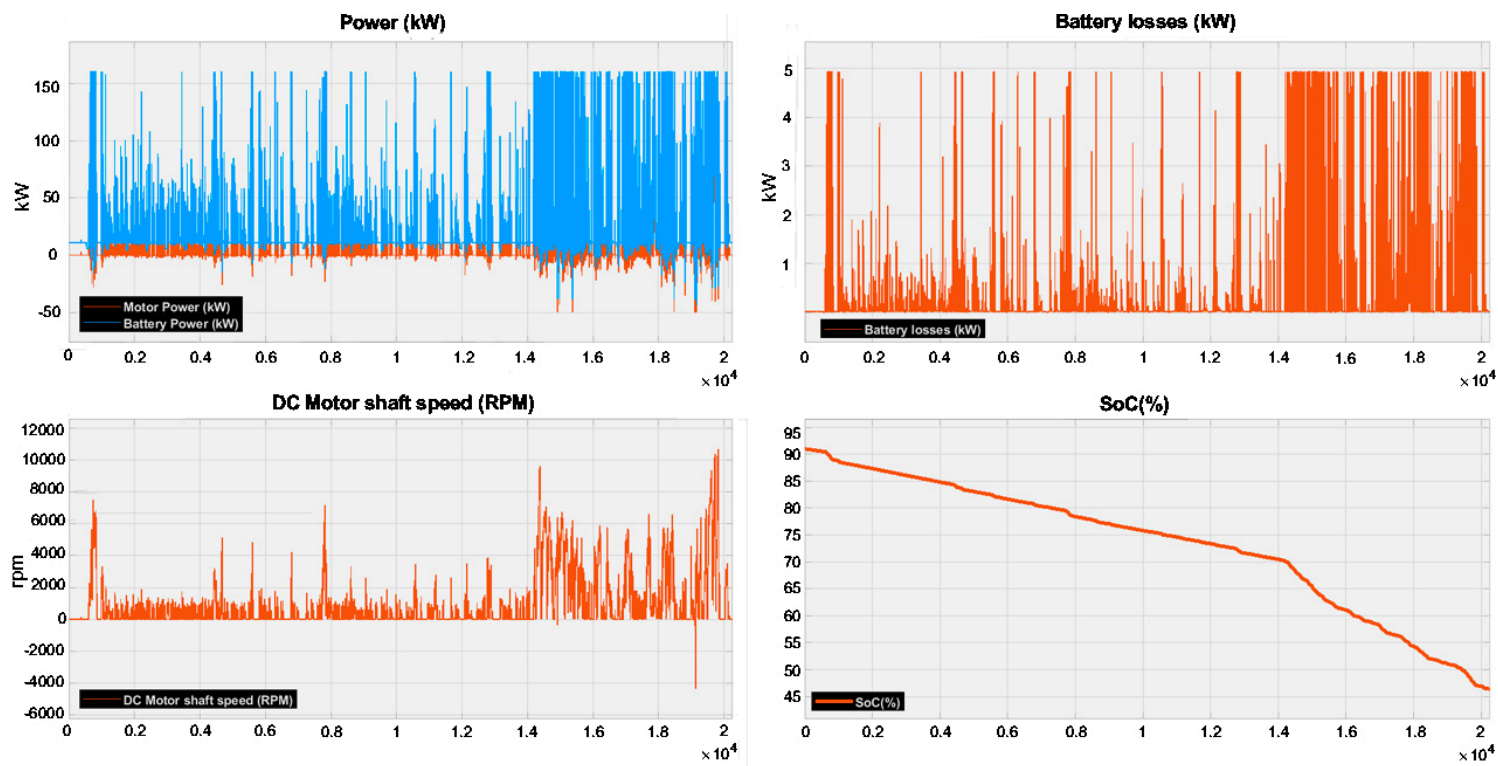

Figure 4. Power information output. Model output example. Top left-power at motor and battery $(\mathrm{kW})$, Top Right—battery losses $(\mathrm{kW})$, Bottom Left—motor RPM, Bottom Right—SoC.

To ensure a reasonable degree of precision while maintaining functionality and flexibility during the simulation, the model employs commonly used measurement and control techniques that have been tailored for a given set of physical constraints. Vehicle response to the input dataset is achieved using proportional-integral-derivative (PID) control assisted by further mathematical system dampening, as presented in Figure 5. PID values were chosen initially using the built-in MATLAB control response tuning system, then fine-tuned by trial and error. An algebraic constant was connected to the PID controller output to avoid solution convergence problems. 


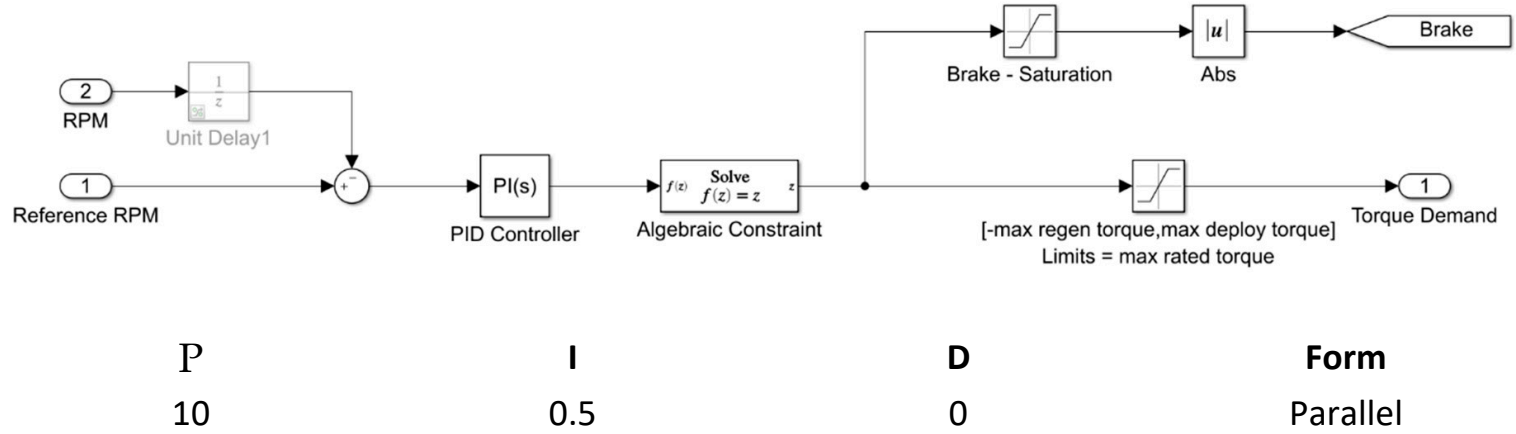

Figure 5. Control module system design and proportional-integral-derivative (PID) controller main specifications.

Similarly, the proposed model showcases quick system diagnosis methods at a system-block level through conventional Simulink monitoring, but also employing the SimScape results explorer module. Finally, performance assessments of all of the existing mathematical solvers feasible for the given model were performed in order to choose the best-suited model for the application.

\section{Evaluation}

The proposed driving route simulation algorithm and the energy consumption model were evaluated using data randomly sampled from the collection rota mentioned above. The sample used for evaluation is plotted in Figure 6.

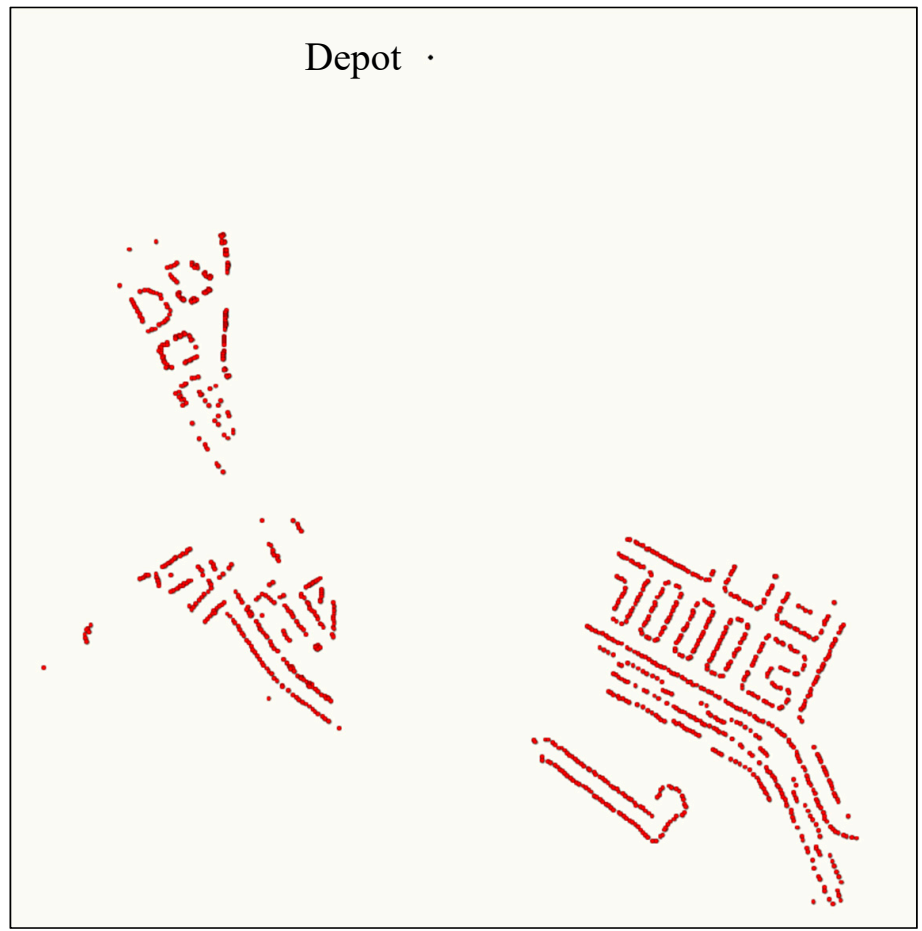

Figure 6. The plot of the sample data used for evaluation.

Utilising the algorithm presented above, a simulated virtual drive path was created and is shown in Figure 7. As can be seen from the figure, the proposed algorithm successfully generated a validated virtual driving path that covers all desired addresses while ensuring repeated sections are at a minimum. 


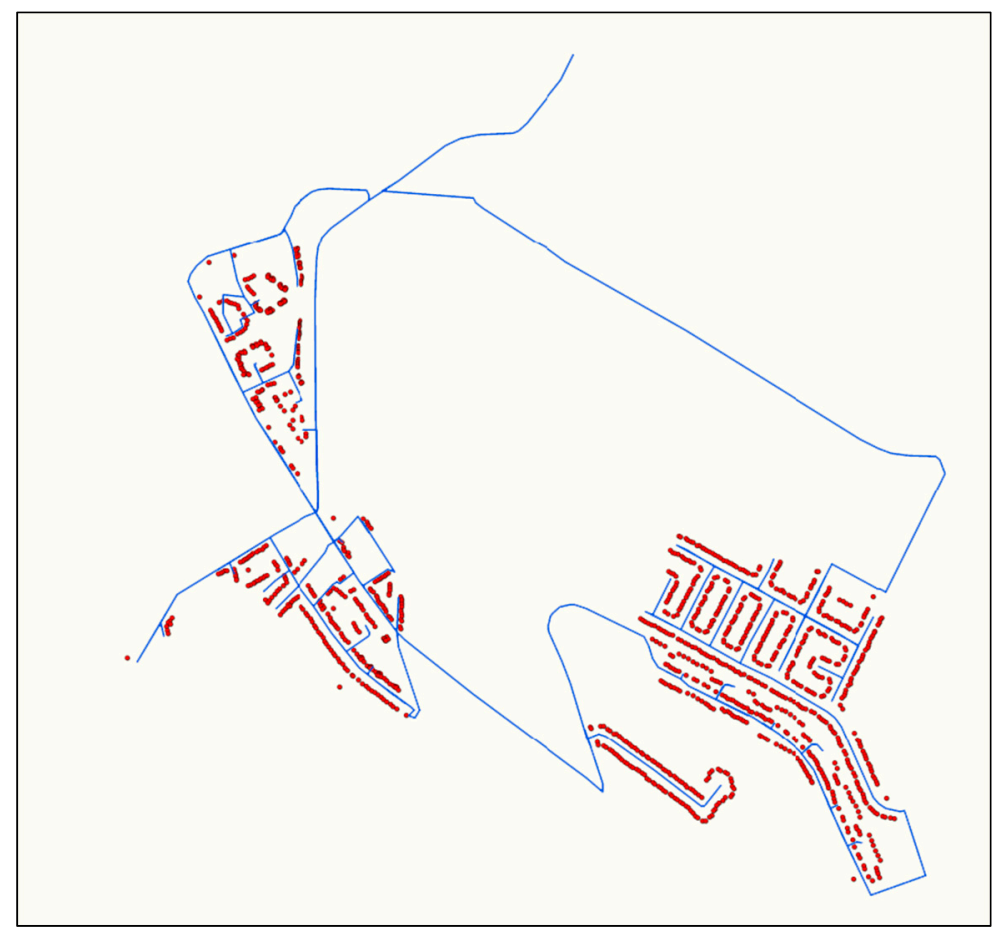

Figure 7. Simulated virtual driving route for the select sample address set, utilising a given road structure.

Figure 8 shows a comparison between the simulated virtual driving route and driving route collected from a real vehicle using a Global Positioning System (GPS)-based logging device. As can be seen from the figure, a negligible difference can be found between these routes, demonstrating the efficacy of the proposed route simulation algorithm.

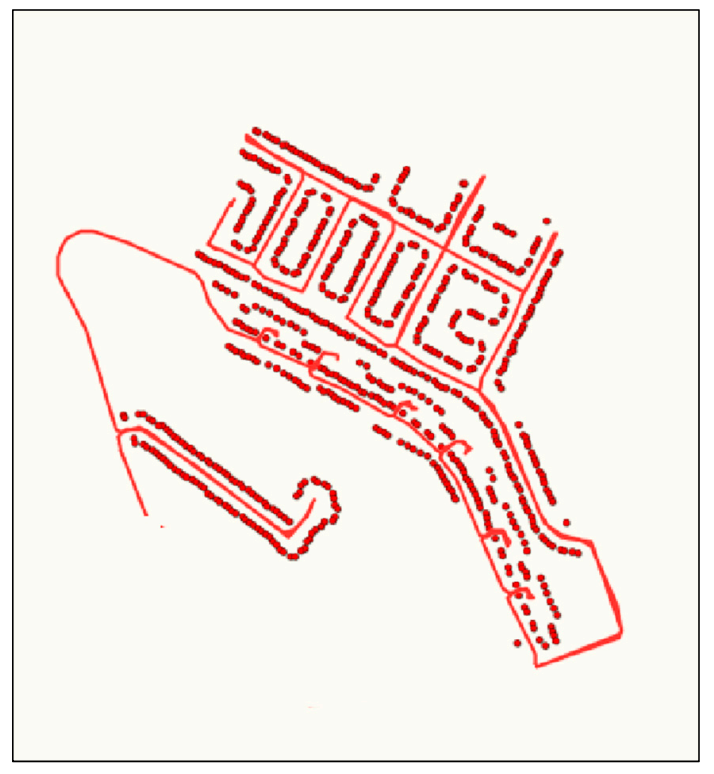

(a)

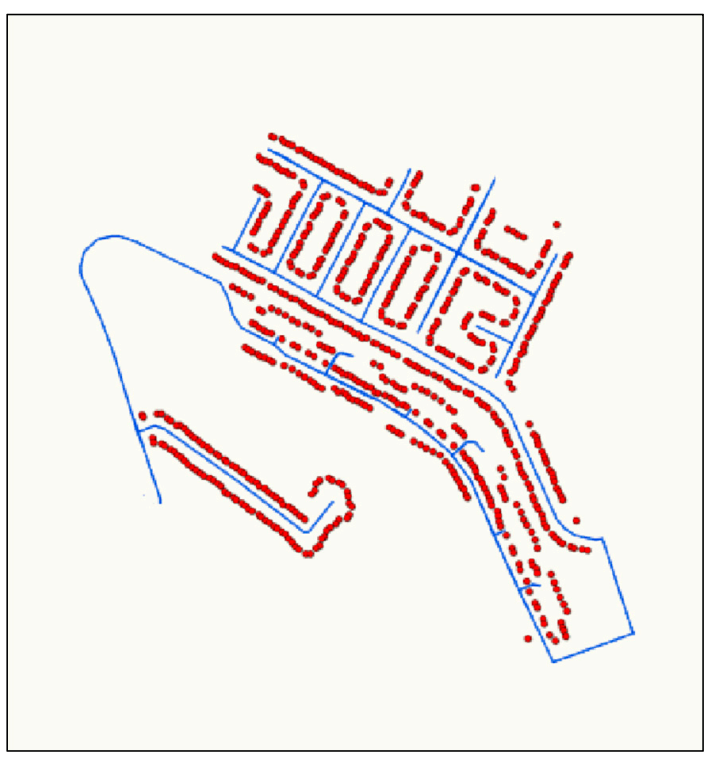

(b)

Figure 8. Comparison between real (a) and simulated (b) driving route.

From the simulated virtual driving route shown above, the corresponding speed profile was generated using the parameters shown in Table 2. 
Table 2. Parameters used in trapezoidal approximation.

\begin{tabular}{cc}
\hline Parameter & Value \\
\hline Maximum distance between two clusters of bins & $25 \mathrm{~m}$ \\
Maximum speed during cruise & $30 \mathrm{~km} / \mathrm{h}$ \\
Maximum speed during collection & $12.6 \mathrm{~km} / \mathrm{h}$ \\
Stop time for drivers to empty bins & $10 \mathrm{~s}$ \\
Positive slope for acceleration & $0.3522 \mathrm{~m} / \mathrm{s}^{2}$ \\
Negative slope for deceleration & $0.3057 \mathrm{~m} / \mathrm{s}^{2}$ \\
Stop time at traffic lights & $38 \mathrm{~s}$ \\
\hline
\end{tabular}

As can be seen from Figure 9a, the speed profile can be split into cruise sections and collection sections based on its speed characteristics, corresponding to real-world RCVs which tend to drive at a higher speed during cruise sections and operate at a significantly lower speed when collecting bins. Figure $9 b, c$ shows the speed profile during collection.

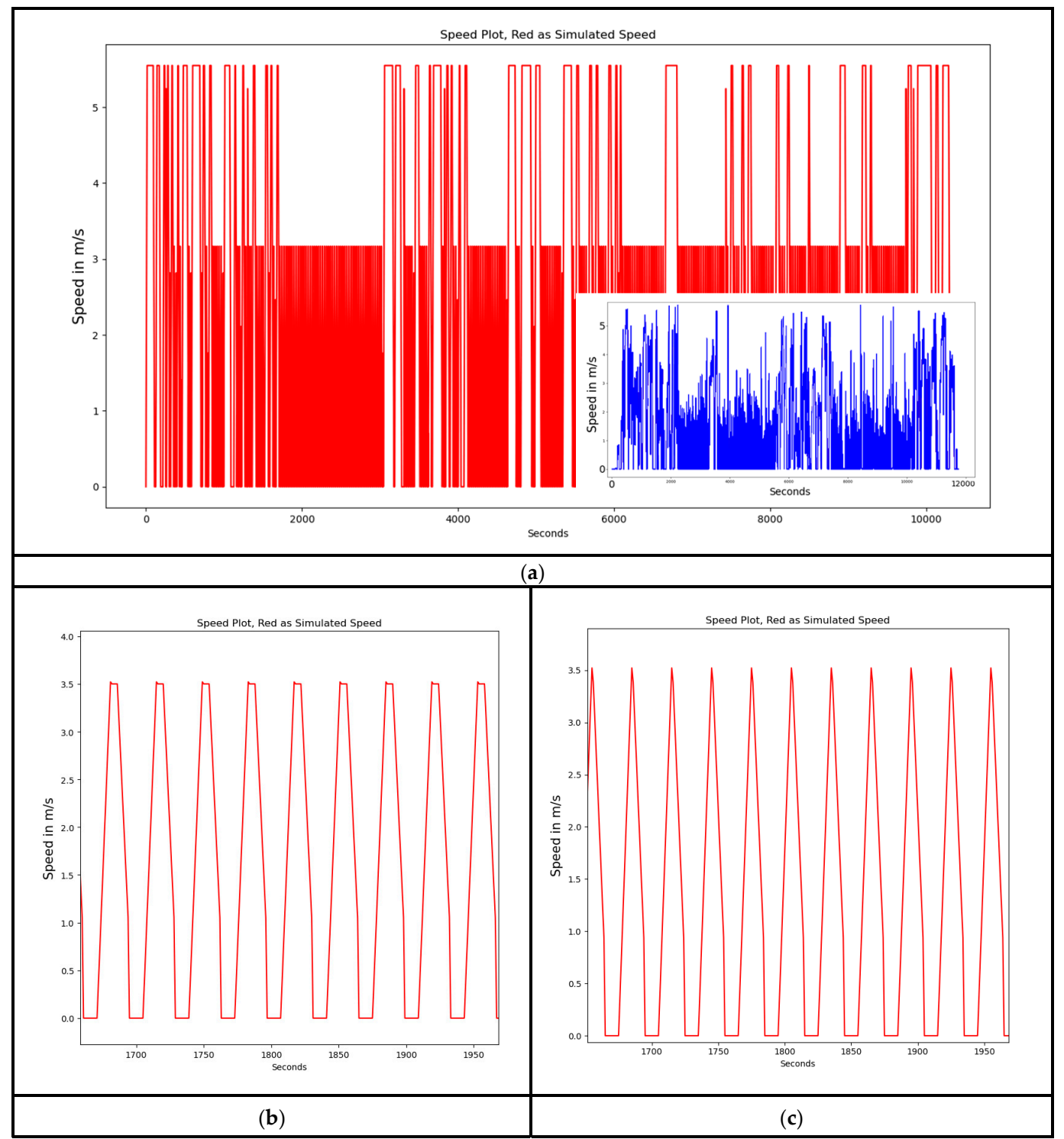

Figure 9. (a) Simulated speed profile with an embedded blue figure showing the corresponding real-life speed profile, (b) speed profile during collection with maximum distance between clusters of bins set to $50 \mathrm{~m}$, (c) speed profile during collection with maximum distance between clusters of bins set to $25 \mathrm{~m}$. 
Figure $9 \mathrm{~b}$ illustrates the four speed states of the proposed trapezoidal speed approximation: acceleration, constant speed, deceleration and parking for moving bins. When the maximum distance between two clusters of bins is set to a smaller value where pre-defined maximum collection speed $(12.6 \mathrm{~km} / \mathrm{h})$ cannot be achieved, a triangle-like speed profile is obtained. The speed profile of such a configuration is shown in Figure 9c.

Next, the energy consumption model was also evaluated using real-life data, but in two stages. First, real-life driving routes were used to evaluate the efficacy of the proposed modelling methodology. The results obtained from the proposed model were compared with the real-life SoC figure collected together with the driving route. During the evaluation, driving cycles were chosen at random from provided datasets with behaviour observed for over 130 kilometres of driving cycles in total. Throughout this evaluation stage, the average error rate (i.e., the difference in SoC percentage at the end of the simulation between real-life data and simulation) was calculated to be $6.08 \%$. The figure drops to $5.8 \%$ if adjusting for outlier marker attenuation. A comparative histogram, using data from simulated results and real-life data logged from trialling eRCVs, showcasing several of these error rates per cycle, is presented in Figure 10. The chosen driving cycles represent an average rubbish collection route, with a mix of driving features, including intense acceleration, start-stop phases as well as sustained speed (cruising). While some minor differences can be seen from the figure, they did not significantly affect the end result in terms of power usage, maintaining a high degree of dependability.

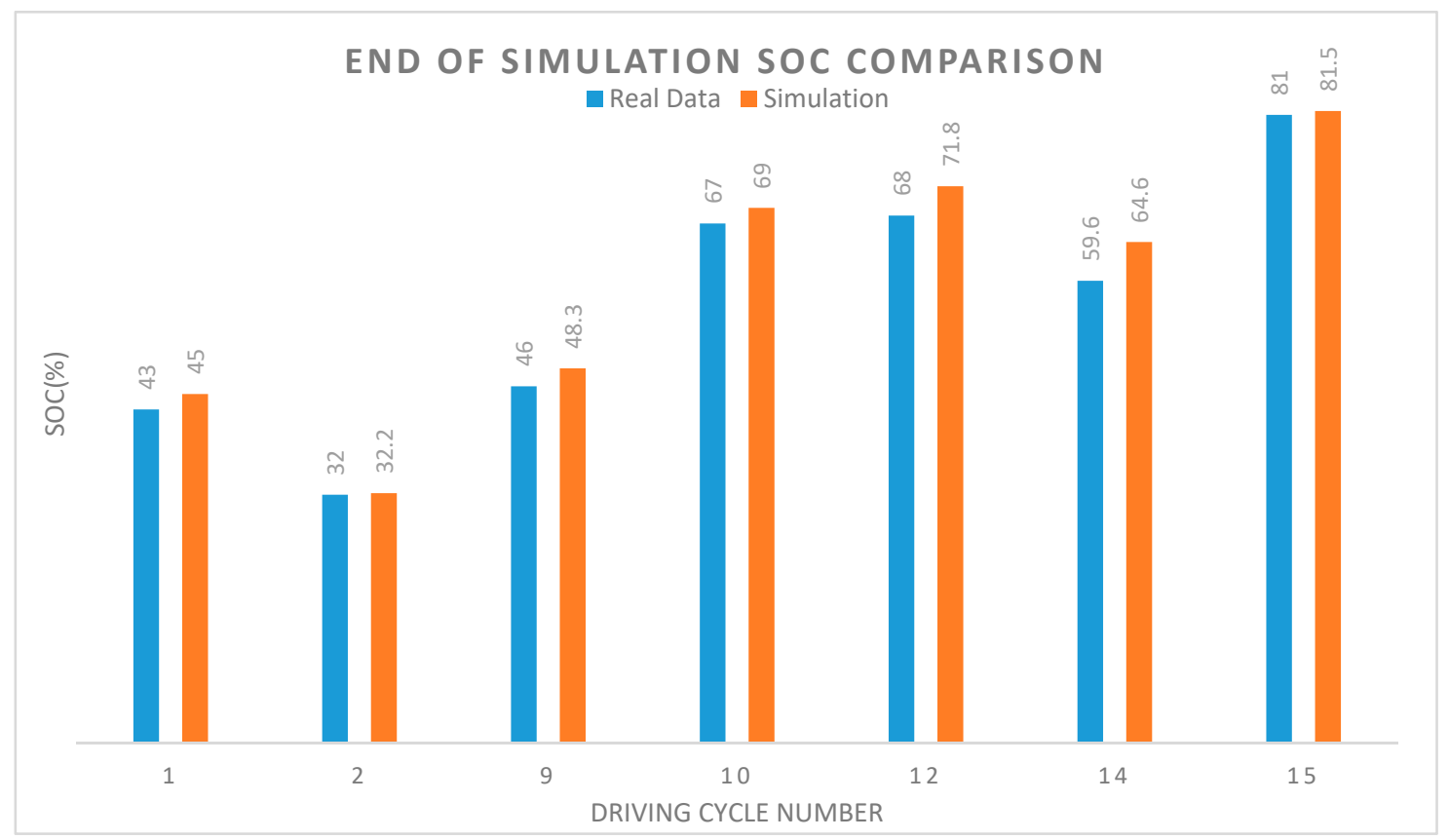

Figure 10. Comparison between real-life and simulated end-of-collection SoC using a real-life driving route.

In order to validate the effectiveness of the proposed address-driven energy prediction methodology, the integrated system was evaluated during the late development phase. This evaluation was primarily focused on the bin-collection stage, where the eRCV uses most of its energy. As can be seen from Figure 10, a simulated drive route for each bin-collection stage was generated and fed into the model. Results were compared between the real-life energy usage and the energy usage calculated from the generated drive route. It can be seen from Figure 11 that the average additional error rate in SoC prediction was $2.64 \%$. 


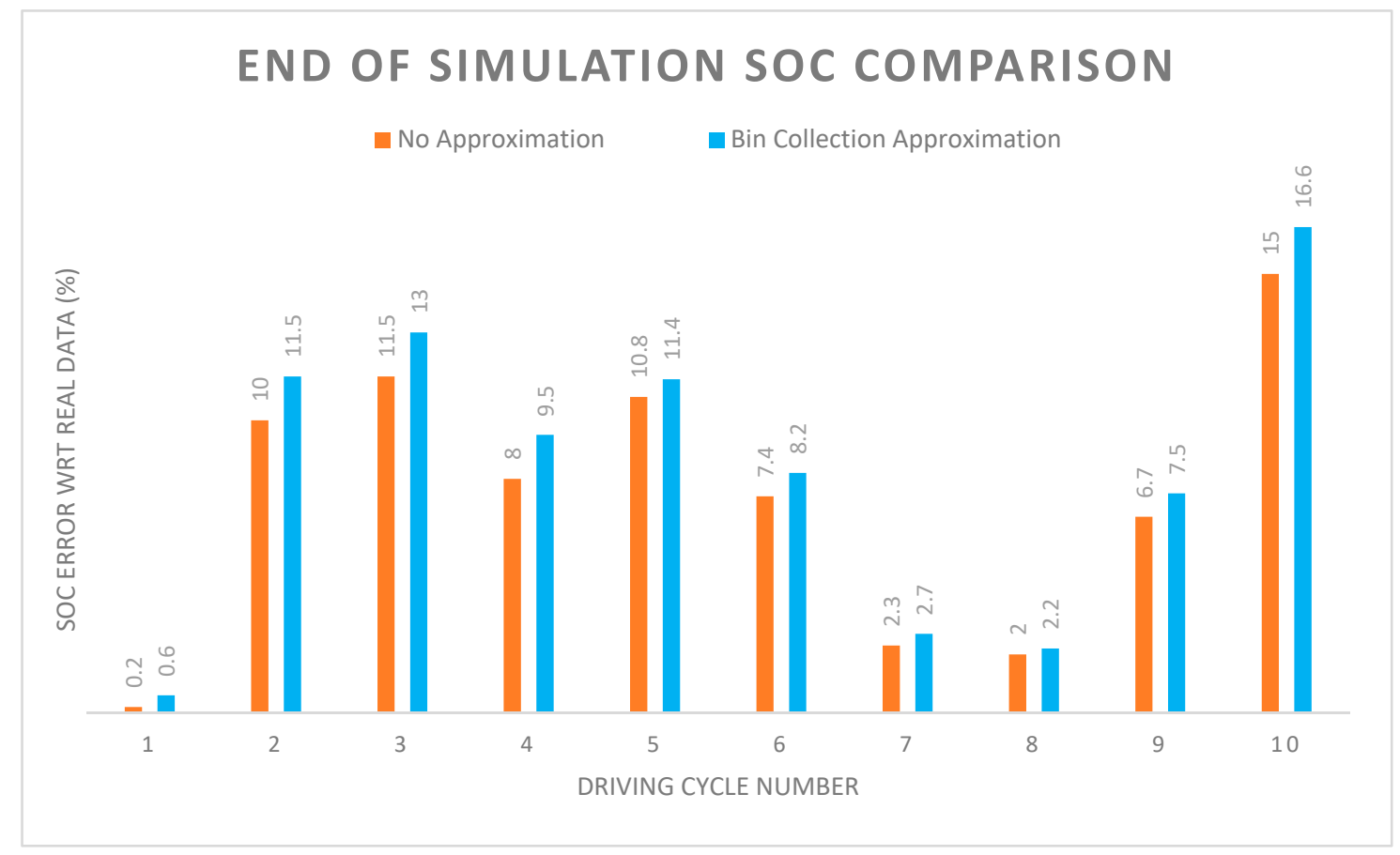

Figure 11. Comparison between real-life and simulated end-of-collection SoC using simulated driving route.

As can be seen from the above evaluation, the results successfully demonstrate the consistency and accuracy of the proposed drive route simulation algorithm and energy prediction model, thus validating the efficacy of the proposed address-driven energy prediction methodology.

\section{Conclusions}

Municipal services such as refuse collection play a vital role in contemporary communities and require an increasing number of vehicles. To reduce noise pollution and GHG emission, eRCVs have been proposed as replacements to the polluting diesel-powered rubbish collection vehicles. However, the energy storage component, primarily the chemical battery of the eRCV, has a unique feature compared with the conventional energy storage compartment, such as the fuel tank, where the battery maintains the same weight independent of its SoC. Research has shown that the battery contributes the majority of the vehicle's kerb weight, and thus carrying an oversized battery could crucially jeopardise the significance of the eRCV in terms of efficiency and cost.

This paper introduces a methodology for predicting the amount of energy required to cover a set of addresses by implementing higher proportions of route planning and energy modelling schemes. The detailed algorithm for route building and comprehensive theory of modelling for energy calculation is presented. The methodology proposed in this paper was evaluated using randomly sampled data from in-service collection schedules in the UK. The results obtained from the evaluation phase successfully validated the proposed algorithm and energy model.

Based on the validated model, a set of calculations for each planned route can be processed. The cluster of energy figures obtained will illustrate the energy usage, and thus battery capacity requirements, for each given route. Therefore, optimisation of the onboard battery capacity for a designated eRCV fleet can be achieved, reducing the battery weight to the maximum degree and achieving a further level of sustainability. The proposed energy prediction methodology could enable municipal services to further reduce their energy usage and establish more sustainable smart city space, services and structures. 
Author Contributions: Conceptualization, Funding acquisition, Project administration, Resources, Supervision, E.E.F.B. and D.A.S.; Data curation, Formal analysis, Investigation, Writing-review and editing, R.Z., T.S., E.E.F.B. and D.A.S.; Methodology, Validation, R.Z., T.S. and D.S.; Writing-original draft, R.Z. and T.S. All authors have read and agreed to the published version of the manuscript.

Funding: This research was funded by EPSRC IAA (University of Sheffield Knowledge Exchange fund).

Acknowledgments: The authors wish to thank all those that have generously provided industry and local authority insights and participated in the research.

Conflicts of Interest: The authors declare no conflict of interest.

$\begin{array}{ll}\text { Abbreviations } \\ \text { The following abbreviations are used in this manuscript: } \\ \text { eRCV } \quad \text { electric refuse collection vehicle } \\ \text { RCV } \quad \text { refuse collection vehicles } \\ \text { SoC } & \text { state of charge } \\ \text { GHG } & \text { greenhouse gas } \\ \text { HGV } & \text { heavy goods vehicle } \\ \text { CNG } & \text { compressed natural gas } \\ \text { ICE } & \text { internal combustion engine } \\ \text { EV } & \text { electric vehicle } \\ \text { PSO } & \text { particle swarm optimisation } \\ \text { NLP } & \text { natural language processing } \\ \text { GIS } & \text { geographic information system } \\ \text { BEV } & \text { battery-electric vehicle } \\ \text { DC } & \text { direct current } \\ \text { RPM } & \text { revolutions per minute } \\ \text { PID } & \text { proportional-integral-derivative } \\ \text { GPS } & \text { Global Positioning System }\end{array}$

\section{References}

1. 2019 UK Greenhouse Gas Emissions Provisional Figures. Available online: https://assets.publishing.service. gov.uk/government/uploads/system/uploads/attachment_data/file/875485/2019_UK_greenhouse_gas_ emissions_provisional_figures_statistical_release.pdf (accessed on 27 August 2020).

2. Vehicle Licensing Statistics. 2019. Available online: http://www.gov.uk/government/statistics/vehiclelicensing-statistics-2019 (accessed on 27 August 2020).

3. UK Statistics on Waste. Available online: https://assets.publishing.service.gov.uk/government/uploads/ system/uploads/attachment_data/file/918270/UK_Statistics_on_Waste_statistical_notice_March_2020_ accessible_FINAL_updated_size_12.pdf (accessed on 27 August 2020).

4. UK HGV Market Declines in 2017 but Demand for Artics and Refuse Trucks Bucks Trend. Available online: http://www.smmt.co.uk/2018/02/uk-hgv-market-declines-2017-demand-artics-refusetrucks-bucks-trend (accessed on 27 August 2020).

5. Lai, C.S.; Jia, Y.; Dong, Z.; Wang, D.; Tao, Y.; Lai, Q.H.; Wong, R.T.; Zobaa, A.F.; Wu, R.; Lai, L.L. A Review of Technical Standards for Smart Cities. Clean Technol. 2020, 2, 290-310. [CrossRef]

6. U.K. Adopts Law for Net Zero Fossil Fuel Pollution by 2050. Available online: www.bloomberg.com/news/ articles/2019-06-24/u-k-adopts-law-for-net-zero-fossil-fuel-pollution-by-2050 (accessed on 27 August 2020).

7. U.S. Department of Energy. Clean Cities Niche Market Overview: Refuse Haulers. 2011. Available online: https://afdc.energy.gov/files/pdfs/51588.pdf (accessed on 27 August 2020).

8. Agreement, P. Paris Agreement. In Report of the Conference of the Parties to the United Nations Framework Convention on Climate Change (21st Session, 2015: Paris); United Nations: Paris, France, 2015; Volume 4, p. 2017.

9. Quiet Revolution in Trash Trucks. Available online: https://www.govtech.com/transportation/QuietRevolution-in-Trash-Trucks.htm (accessed on 27 August 2020).

10. Zhao, Y.; Tatari, O. Carbon and energy footprints of refuse collection trucks: A hybrid life cycle evaluation. Sustain. Prod. Consum. 2017, 12, 180-192. [CrossRef] 
11. Shindell, D.T.; Faluvegi, G.; Koch, D.M.; Schmidt, G.A.; Unger, N.; Bauer, S.E. Improved attribution of climate forcing to emissions. Science 2009, 326, 716-718. [CrossRef] [PubMed]

12. Case Study-Compressed Natural Gas Refuse Fleets. Available online: https://afdc.energy.gov/files/u/ publication/casestudy_cng_refuse_feb2014.pdf (accessed on 27 August 2020).

13. Canter, N. Hybrid hydraulic drive reaps fuel savings. Tribol. Lubr. Technol. 2008, 64, 14.

14. Bringing Electric Trucks to New Zealand. Available online: http://Pureadvantage.org/news/2017/02/02/ bringing-electric-trucks-new-zealand (accessed on 27 August 2020).

15. UK Petrol Prices Rise to Three-Year High. Available online: www.independent.co.uk/news/business/ news/uk-petrol-prices-high-three-years-unleaded-per-litre-motorists-car-costs-a8140066.html (accessed on 27 August 2020).

16. DECC, D. Fossil Fuel Price Projections, July 2013 (URN 13D/170); Department of Energy and Climate Change (DECC): London, UK, 2013.

17. RAC Warns Good Times of Lower Cost Fuel Appear to be over as Prices Reach Three Year High. Available online: http://www.telegraph.co.uk/news/2018/01/04/rac-warns-good-times-lower-cost-fuelappear-prices-reach-three (accessed on 27 August 2020).

18. RL, V.K.; Tripathy, B.; Radhakrishnan, J. Simulation of Softening and Rupture in Multilayered Fuel Tank Material (No. 2019-28-2557); SAE Technical Paper; SAE: Warrendale, PA, USA, 2019.

19. Wang, Q.; Mao, B.; Stoliarov, S.I.; Sun, J. A review of lithium ion battery failure mechanisms and fire prevention strategies. Prog. Energy Combust. Sci. 2019, 73, 95-131. [CrossRef]

20. Arora, S.; Shen, W.; Kapoor, A. Review of mechanical design and strategic placement technique of a robust battery pack for electric vehicles. Renew. Sustain. Energy Rev. 2016, 60, 1319-1331. [CrossRef]

21. Dynamometer Drive Schedules. Available online: http://www.epa.gov/vehicle-and-fuel-emissions-testing/ dynamometer-drive-schedules (accessed on 27 August 2020).

22. Brundell-Freij, K.; Ericsson, E. Influence of street characteristics, driver category and car performance on urban driving patterns. Transp. Res. Part D 2005, 10, 213-229. [CrossRef]

23. Chen, Y.; Nakazawa, J.; Yonezawa, T.; Tokuda, H. Cruisers: An automotive sensing platform for smart cities using door-to-door garbage collecting trucks. Ad. Hoc. Netw. 2019, 85, 32-45. [CrossRef]

24. Read, A.D. Where There's Muck There's Brass: The'Cost'of London's Waste? Area 2001, 33, 103-106.

25. The Case for Repowering Refuse Collection Vehicles from Diesel to Electric. Available online: http://static1.squarespace.com/static/5ae87244b105982d79d73cf5/t/5be2dbac0e2e72e2e81ba15c/ 1541594076246/ERCV+Final+Report.pdf (accessed on 27 August 2020).

26. Petroleum Vapor Intrusion: Fundamentals of Screening. Available online: http://www.itrcweb.org/Team/ Public?teamID=12 (accessed on 27 August 2020).

27. Health and Exposure to Air Pollution. Available online: http://www.london.gov.uk/what-we-do/ environment/pollution-and-air-quality/health-and-exposure-pollution (accessed on 27 August 2020).

28. Plans for UK's First Fully Electric Refuse Fleet as Veolia Signs New Tech-Driven City Waste Contract. Available online: http://News.cityoflondon.gov.uk/plans-for-uks-first-fully (accessed on 27 August 2020).

29. Scottish Firm Latest to Trial Electric RCVs. Available online: http://www.mrw.co.uk/latest/scottish-firmlatest-to-trial-electric-rcvs-02-10-2018 (accessed on 27 August 2020).

30. Veolia to Trial Electric Refuse Collection Vehicles. Available online: http://www.veolia.co.uk/press-releases/ veolia-trial-electric-refuse-collection-vehicles (accessed on 27 August 2020).

31. Electric Refuse Collection Vehicle Project. Available online: https://www.digitalgreenwich.com/electricrefuse-collection-vehicle-project (accessed on 27 August 2020).

32. Battery State of Charge Determination. Available online: https://www.mpoweruk.com/soc.htm (accessed on 27 August 2020).

33. Xu, G.; Li, W.; Xu, K.; Song, Z. An intelligent regenerative braking strategy for electric vehicles. Energies 2011, 4, 1461-1477. [CrossRef]

34. Hong, Z.; Xu, L.; Min, W. Adaptive Kalman filter based state of charge estimation algorithm for lithium-ion battery. Chin. Phys. B 2015, 24, 098801.

35. Abousleiman, R.; Rawashdeh, O. A Bellman-Ford approach to energy efficient routing of electric vehicles. In Proceedings of the 2015 IEEE Transportation Electrification Conference and Expo (ITEC), Dearborn, MI, USA, 14-17 June 2015; pp. 1-4. 
36. Abousleiman, R.; Rawashdeh, O. April. Energy-efficient routing for electric vehicles using metaheuristic optimization frameworks. In Proceedings of the MELECON 2014-2014 17th IEEE Mediterranean Electrotechnical Conference, Beirut, Lebanon, 13-16 April 2014; pp. 298-304.

37. Abousleiman, R.; Rawashdeh, O. Electric vehicle modelling and energy-efficient routing using particle swarm optimisation. IET Intell. Transp. Syst. 2016, 10, 65-72. [CrossRef]

38. An Application of ant Colony Optimization to Energy Efficient Routing for Electric Vehicles. Available online: http://www.sae.org/publications/technical-papers/content/2013-01-0337 (accessed on 27 August 2020).

39. Abousleiman, R.; Rawashdeh, O. Tabu search based solution to the electric vehicle energy efficient routing problem. In Proceedings of the 2014 IEEE Transportation Electrification Conference and Expo (ITEC), Dearborn, MI, USA, 15-18 June 2014; pp. 1-6.

(C) 2020 by the authors. Licensee MDPI, Basel, Switzerland. This article is an open access article distributed under the terms and conditions of the Creative Commons Attribution (CC BY) license (http://creativecommons.org/licenses/by/4.0/). 